\title{
Force-dependent cell signaling in stem cell differentiation
}

\author{
Evelyn KF Yimm ${ }^{1,2,3}$ and Michael P Sheetz ${ }^{* 1,4}$
}

\begin{abstract}
Stem cells interact with biochemical and biophysical signals in their extracellular environment. The biophysical signals are transduced to the stem cells either through the underlying extracellular matrix or externally applied forces. Increasing evidence has shown that these biophysical cues such as substrate stiffness and topography can direct stem cell differentiation and determine the cell fate. The mechanism of the biophysically induced differentiation is not understood; however, several key signaling components have been demonstrated to be involved in the force-mediated differentiation. This review will focus on focal adhesions, cytoskeletal contractility, Rho GTPase signaling and nuclear regulation in connection with biophysically induced differentiation. We will briefly introduce the important components of the mechanotransduction machinery, and the recent developments in the study of force-dependent stem cell differentiation.
\end{abstract}

In the stem cell niche, stem cells receive biochemical and biophysical signals, which dictate the cell fate in development or regeneration. These cues have been demonstrated to be crucial in directing stem cell differentiation in numerous studies. Stem cell differentiation using biochemical cues, such as growth factors or small molecule inhibitors, has been extensively studied. Meanwhile, inducing stem cell differentiation using biophysical signals is not well understood and has recently been attracting attention. Breakthroughs in research have highlighted the significance of mechanotransduction in stem cell differentiation. Biophysical signals can be transduced into changes in cell biochemistry through

*Correspondence: mbihead@nus.edu.sg

'Mechanobiology Institute Singapore, National University of Singapore, T-Lab,

\#05-01, 5A Engineering Drive 1, Singapore 117411, Singapore

Full list of author information is available at the end of the article mechanotransduction of interactions with the extracellular matrix (ECM) substrate, or interactions with neighboring cells; in specific cases, fluid flow forces are also involved in altering cell biochemistry. Examples of these biophysical cues are illustrated in Figure 1. Biophysical cues can be controlled by manipulating substrate stiffness [1], by ECM patterning techniques to confine cell shape [2], by modifying surface topography [3,4], or by applying external forces (compressive, tensile or shear force) [5].

Mechanotransduction involves a complex interplay of different cellular organelles and components, which by themselves are highly dynamic in vivo [6]. However, advancement in experimental techniques has helped to provide increasing evidence about the mechanisms and the cellular components such as the integrins, focal adhesions (FAs) and cytoskeleton organization that collectively play important roles in biophysically induced cellular behaviors [6-8].

Nonetheless, the underlying mechanism of biophysically induced stem cell differentiation remains unclear. Collective research efforts have revealed several key signaling components involved in mechanically induced differentiation. While various indirect and physiochemical mechanisms such as calcium signaling [9] and mechanosensitive ion channels [10] have been studied, in this review we will focus on FAs and cytoskeletal contractility, Rho GTPase signaling and nuclear regulation. We will briefly introduce the important components of the mechanotransduction machinery, and the recent discoveries in force-dependent stem cell differentiation. The general overview of mechanical control of stem cell differentiation has been discussed in a few excellent reviews [5,11-13]. For details of mechanotransduction in cell regulation, readers can refer to reviews by Vogel and Sheetz $[14,15]$.

\section{Integrins and focal adhesions: inside out and outside in}

Anchorage-dependent cells are able to adhere to the underlying ECM substrate by employing membranebound integrins. Various different types of adhesions exist between cells and the ECM, and they perform 


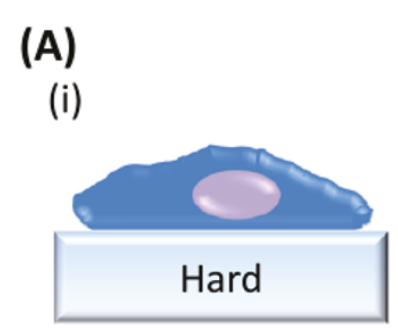

(ii)

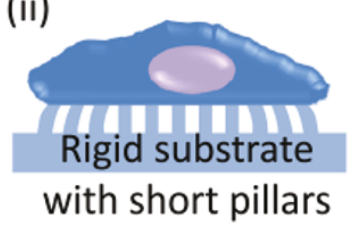

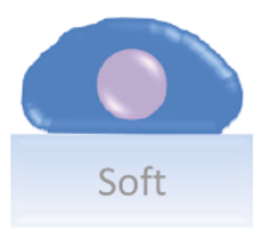

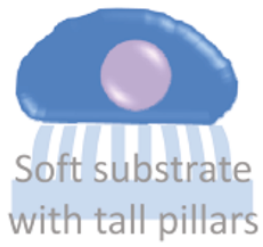

(B)

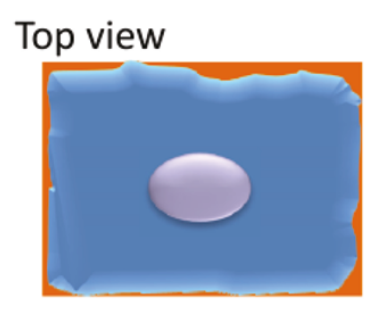

Side view

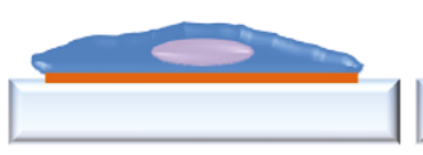

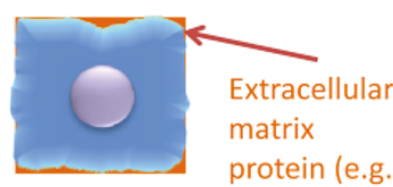

fibronectin)

printed

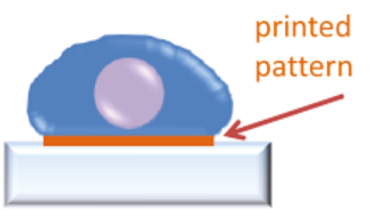

(C)

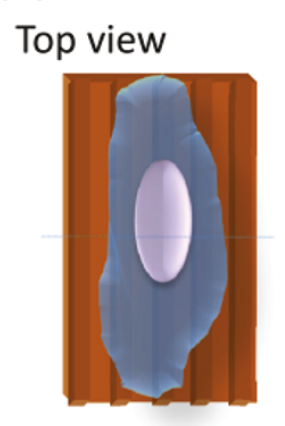

Side view

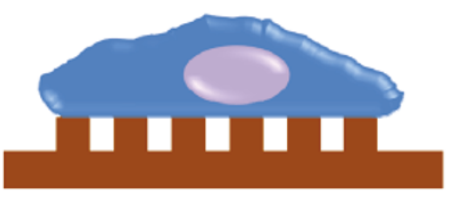

(D)

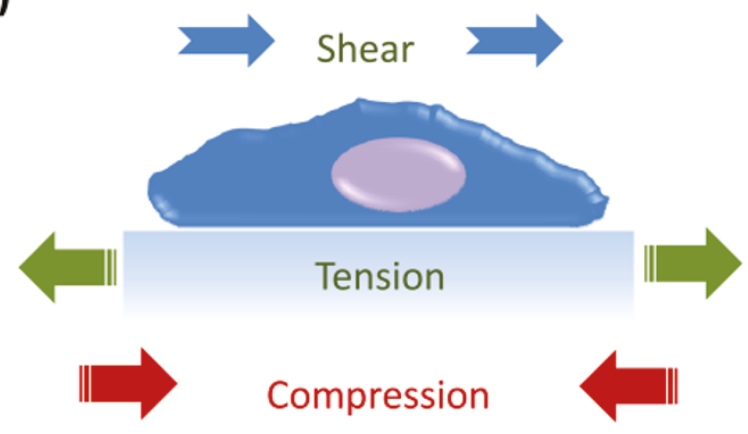

Figure 1. Schematic diagrams of various methods to apply biophysical signals to cells. (A) Manipulation of substrate stiffness by (i) tuning the rigidity of the materials or (ii) using micro-post array with different height to adjust the rigidity. (B) Confinement of cell shape by patterning extracellular matrix protein such as fibronectin onto the substrate. Area outside the patterned shape will be coated with anti-fouling reagent to prevent cell adhesion. (C) Modification of surface topography. (D) Application of external forces, such as compression by compressing the substrate, tension by stretching the substrate, or shear stress by fluid flow.

different and specific functions in cells [16]. These types include cell-cell adhesions (for example, cadherins) and cell-matrix interactions. The cell-matrix interactions through integrin-based adhesion complexes [6] are the most fundamental adhesions involved in the stem cells' response to biophysical signals.

Integrins are heterodimeric transmembrane cell adhesion proteins that bind to specific motifs present on the ECM $[17,18]$. Changes in the physical structure of the underlying substrate can influence the clustering of integrins and other adhesion molecules, in turn activating signaling pathways that will ultimately result in modification of cell behavior. Using precise nanoscale adhesive islands, Arnold and colleagues established a relationship among integrin clustering, FA formation and actin stress fibers that influenced the adhesion and spreading of cells [19]. A separation of $\geq 73 \mathrm{~nm}$ between RGD ligand, which is an integrin binding site found in fibronectin, on the ECM substrate will reduce the cell attachment, cell spreading and FA formation, showing the importance of integrin clustering in the regulation of integrin-mediated signal transduction [19]. Further studies have determined that the minimum cluster size is 3 to 4 RGD ligands to produce a similar cluster of integrins [20]. Using a novel nanoscale ligand spacing gradient, Arnold and colleagues also suggested that cells were sensitive to changes in interparticle spacing of about $1 \mathrm{~nm}$ over a cell length, demonstrating the sensitivity of the cellular sensing mechanism [21]. The sensitivity to minute variations may have physiological implications. For example, ECM collagen fibers have a $67 \mathrm{~nm}$ banding periodicity [22] and fibronectin fibers present nanoscale epitopes [23,24].

Upon binding to the ECM ligands, integrins cluster and activate specific signaling pathways. The biophysical cues, such as nanotopography and substrate rigidity, will modify the activation of integrin clustering, which is probably the initial step in subsequent signal transduction in stem cell genomic regulation. In fact, neural stem cells in the central nervous system appear to have higher levels of $\beta_{1}$-integrins, which act as sensors for the changing 
ECM during embryonic development and adult neurogenesis [25]. This correlation between $\beta_{1}$-integrin expression and neurogenesis further suggests that the modulation of integrin expression is involved in sensing and responding to the biophysical cues and the regulation of stem cell differentiation. However, the role of integrins in gene regulation is complex because these receptors participate in both the sensory and operational functions of the cellular machinery, also commonly known as the outside-in (sensory) and inside-out (operational) signaling activities. The observed dynamics of the integrins on the ECM substrate can be due to both the response of the cell to the underlying ECM and/or a secondary effect of the actin-cytoskeleton FA feedback machinery. The complexity in the feedback network connecting the sensory and operational functions is also reflected in the highly intertwined integrin adhesome network [26].

One of the most important integrin-mediated adhesions involved in mechanotransduction is a FA [6,27], which links the actin cytoskeleton to the transmembrane integrins $[26,28]$. FAs are composed of a large complex network of adhesion molecules [16]. Some of the important structural proteins include talin, vinculin and focal adhesion kinase (FAK) (Figure 2A). The formation and maturation of a FA are driven by feedback between the actin cytoskeleton and integrin [6]. Briefly, talin connections of integrin dimers with the actin filaments are needed for the recruitment of additional components in the complex [29]. The subsequent maturation of the complex requires contractile force to be generated by the actomyosin machinery [30,31]. Readers can refer to an excellent review by Geiger and colleagues for a more detailed description of the steps and mechanism of the FA assembly [6], and to a recent study by Kanchanawong and colleagues for the nanoscale architecture of the FA [32].

The mechanical force exerted on cells plays an important role in the promotion of FA formation. Another important component of FAs, vinculin, triggers the clustering of activated integrins [33]. The binding of vinculin to talin during the initial stages of FA assembly is force mediated, and the binding site for vinculin requires unfolding that is achieved by mechanical forces [34]. Indeed, stretching of the talin molecule can expose binding sites for vinculin, facilitating the recruitment and activation of vinculin at the FA [35]. Similarly for p130cas and fibronectin, mechanical forces can expose cryptic sites for phosphorylation or for interaction with cell surface receptors, respectively $[24,36,37]$.

A recent study using human fibroblasts has demonstrated that cell polarization is matrix rigidity dependent, in which cells are polarized on rigid substrates but not on compliant substrates [38]. Using a systematic siRNAmediated knockdown of 85 human protein tyrosine kinases, changes in substrate rigidity-dependent traction force development and FA mechanosensing are observed to be accompanied by abnormalities in the cell polarization response. The authors therefore propose that the protein tyrosine kinase-dependent molecular checkpoints jointly control cell contractility and FA-mediated mechanosensing.

FA mechanosensing has been shown crucial for stem cells and force-mediated differentiation. In a study of mesenchymal stem cell (MSC) responses to micropillar substrates, FA maturation and actin polymerization were promoted in the MSCs on the micropatterns [39]. By inhibiting the Rho-associated kinase (ROCK) and nonmuscle myosin II, the cytoskeletal contractility, FA formation and FAK activity were reduced. We believe that the FA signal and cytoskeletal contractility were crucial in topography-mediated differentiation. The differentiation of human MSCs will probably correlate with the FAK activity and the cytoskeletal contractility.

The actomyosin contractile stresses that actin exerts on the adhesions are essential for the formation of a FA. The global forces that are experienced by the cells under biophysical cues can alter the forces that the FAs are experiencing, subsequently changing their differentiation lineage. All of these studies indicate that FAs play an important role in mechanotransduction, also in regulating force-induced stem cell differentiation.

\section{Cytoskeletal contractility: testing and responding to the extracellular biophysical environment}

Force generation in the cytoskeleton is required for cell adhesion to the ECM. The contractile cytoskeleton consists of actin, myosin, microtubules and intermediate filaments. They form a network of filamentous proteins that extends throughout the cell cytoplasm in eukaryotic cells. The cytoskeleton has been well studied and an increasing amount of evidence has demonstrated the significance of the cytoskeleton in stem cell differentiation $[1,2,40]$.

Recently, there was a breakthrough in our understanding of the early events in cellular testing of substrate rigidity. As cells spread on substrates, it has been clear that cells sense the rigidity of the matrix materials in a dynamic fashion [41], within the first 2 to 5 minutes upon contact with a surface. Using submicron pillar substrates, it is now clear that local contractions of 1 to $3 \mu \mathrm{m}$ regions of the cell surface are sufficient to sense the substrate rigidity [42]. This observation is consistent with previous studies of matrix-coated beads that showed a minimum cell-substrate contact length of 1 to $2 \mu \mathrm{m}$ was needed to generate cell adhesions [43]. Smaller contact areas developed adhesions only with external force, as applied by laser tweezers. Such local contraction units can only occur if there are anti-parallel actin filaments, which may 


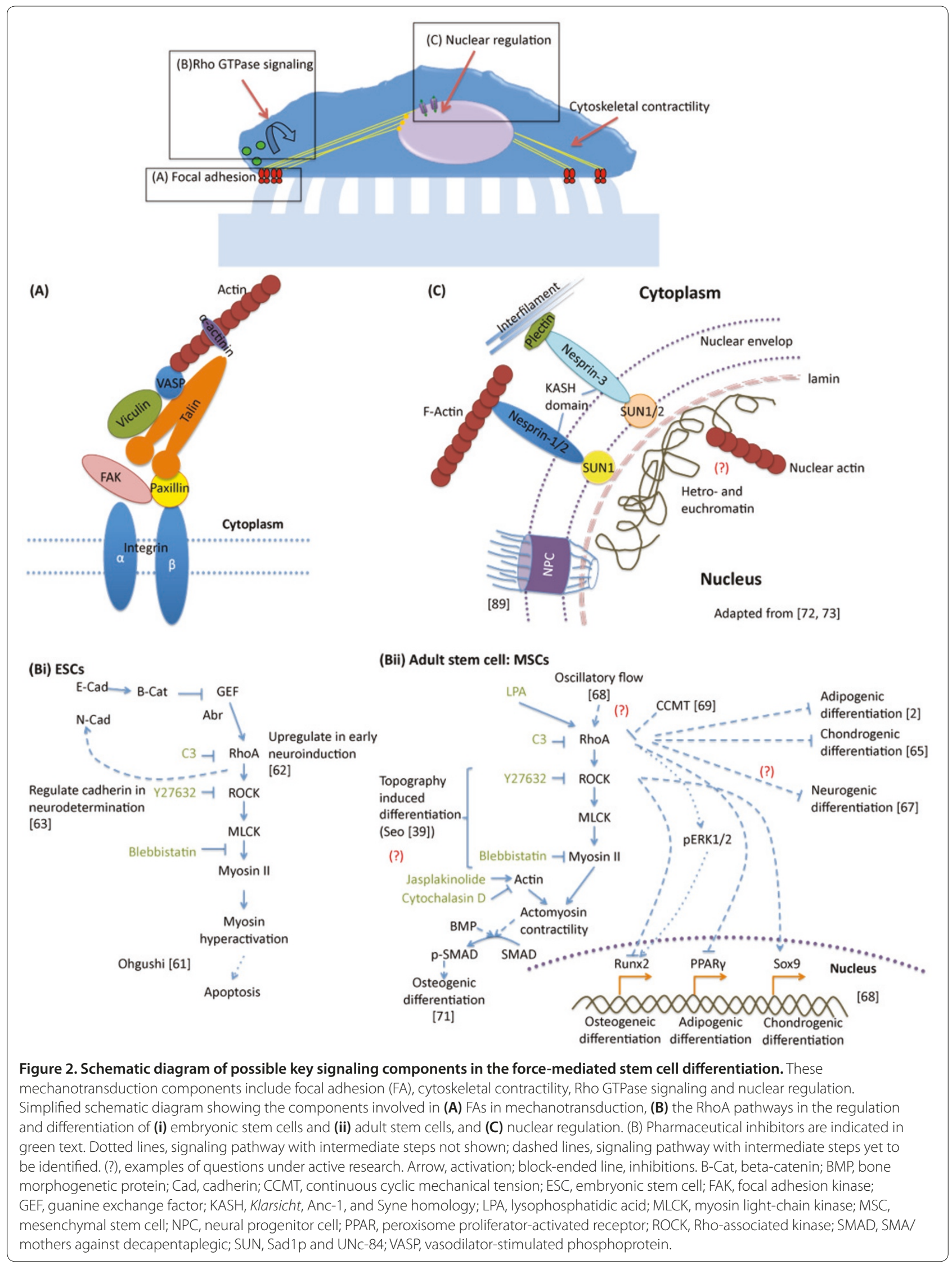


be polymerized from clustered integrins [44]. Since the consequence of rigidity sensing is the development of matrix adhesions, rigidity sensing is only a step in the larger process of mechanosensation and has to be viewed in that larger context.

The contractile forces present in the actin stress fibers of the cytoskeleton are essential in modulating cellular functions. Contractile forces in nonmuscle cells are generated by a class of motor proteins - nonmuscle myosin II. A recent hypothesis suggested that cells use actomyosin contractility for a two-way interaction with the ECM. The cellular response to the biophysical environment is not passive since cells are able to adjust their mechanical properties through the dynamic remodeling of the actin cytoskeleton. The cell contraction through the stress fibers will be resisted by the matrix at the sites of integrin clusters, which will induce the subsequent recruitment of additional molecules for FA formation. The balance of tension forces at these interfacial sites allows the cell to sense the ECM.

In a study by Engler and colleagues, the use of matrices with different elasticity regulates the differentiation of MSCs into different lineages [1]. The use of the specific nonmuscle myosin II inhibitor blebbistatin blocks all elasticity-directed lineage specification without strongly affecting cell function and shape significantly, providing evidence of cytoskeletal force generation in ECM sensing. This tension-mediated signaling is manifested in the reorganization of actin microfilaments or stress fibers to reflect surface features, such as the observed alignment of these stress fibers to nanogratings $[4,7,45]$. This cellular force sensing in turn alters levels of Rho GTPase and mitogen-activated protein kinase activity as downstream biochemical signals for stem cell gene regulation. The correlation between contractile forces in response to substrate rigidity and stem cell differentiation has been further illustrated and supported from other studies in adult stem cells. For example, functional myotubes can be derived from adipose-derived stem cells (ASCs) grown on substrates whose rigidity is similar to that of muscle ECM, and the myotube formation can be manipulated by cytoskeletal contractility [46]. The multi-nucleated myotube fusion can be enhanced using lysophosphatidic acid, which activates Rho and enhances contractility, but is inhibited by the addition of blebbistatin, which inhibits nonmuscle myosin II, in the ASCs. Upon knockdown of $\alpha_{5}$-integrin and $\alpha_{\mathrm{v}}$-integrin, the upregulation of myogenin and MEF2C, which are markers for myogenesis, is abolished [46]. The findings further illustrate that the mechanosensing of substrate rigidity involves cytoskeletal contractility and FA formation in stem cells.

Mechanosensing is also evident in pluripotent stem cells. Human embryonic stem cells (ESCs) are aligned and elongated when they are cultured on nanometer-scale gratings [47]. The cytoskeletal-mediated mechanosensing mechanism seems to be present in human ESCs, whereas mouse ESCs are sensitive to local cyclic stress applied to FAs. The myosin II contractility is essential in mouse ESC stress sensitivity, and the applied stress leads to the downregulation of Oct3/4 gene expression in mouse ESCs [48]. Interestingly, the authors speculated that the sensitivity to local cyclic stress is correlated with the softness of the mouse ESCs.

The softer mouse ESCs, as indicated by lower amounts of F-actin and low actomyosin contractility, appear responsive to cell deformation that can trigger the subsequent spreading [48]. Upon adhesion to the substrate, however, the mouse ESCs did not stiffen when the substrate stiffness increased. The spreading did not further increase on more rigid substrates, but the basal tractions of the mouse ESCs were increased [49]. The stem cells probably interpret such changes in force as signals to regulate stem cell fate. Meanwhile, the cytoskeleton remodeling in the differentiating cells may form a feedback loop, interacting with the biophysical environment.

Stress fibers are constantly tuning the cell's mechanical properties with feedback from its downstream molecules. A growing amount of evidence has demonstrated the importance of actin-myosin contractility $[8,50]$ in mechanotransduction. The cellular contractility induces downstream events including the recruitment of adhesion molecules and kinases such as the mechanosensitive FAK, zyxin and talin, subsequently triggering the activation of Rho GTPases (reviewed in [51] and further discussed below). A similar mechanism is probably important in biophysical-induced stem cell differentiation.

\section{Rho pathway: relaying the mechanical signal and regulating cytoskeletal contractility}

Rho GTPases are molecular switches that control various signal transduction pathways in cells. One of the most well-known roles is regulation of the actin cytoskeleton. (Readers can refer to [52] for a detailed review on RhoGTPase.) Rho, Rac and Cdc42 are the three bestcharacterized members of the Rho family. Many studies illustrate the important role of Rho in the regulation of the actin cytoskeleton in mechanotransduction. Increasing ECM stiffness or force application activates Rho [53]. RhoA then stimulates tension through its effector, Rho kinase, which indirectly elevates the level of the phosphorylated myosin light chain [54]. The Rho activation thus promotes acto-myosin stress fiber assembly [55], significantly changing the mechanical properties of the cell [56].

In human ESCs [57] and other stem cells such as murine prostate stem cells [58], the cells exhibit dissociation-induced apoptosis. This dissociation-induced apoptosis is caused by actomyosin hyperactivation 
through the Rho/ROCK pathway $[59,60]$. The loss of cadherin-dependent intercellular contacts triggers Abr, resulting in an Abr-dependent Rho-high/Rac-low stage [60]. In the presence of a ROCK inhibitor, the survival and cloning efficiency is increased in human ESCs [61] and prostate stem cells [58]. During differentiation, RhoA and Cdc42 RhoGTPase have been observed to be upregulated during early neuroinduction in murine P19 ESCs [62]. The RhoA/ROCK pathway is shown to regulate the cadherin protein level and cell-cell interaction during neurodetermination of the mouse ESCs [63]. Taken together, in human and mouse ESCs, mechanotransduction via the Rho pathway is playing an important role in determining the maintenance of pluripotency, viability, and lineage commitment. The major mechanical signals determining cell fate appear to be triggered by cadherin-dependent cell-cell contact interactions, which interact with and are regulated by the RhoA signals (Figure 2Bi). This phenomenon is different in adult stem cells, in which the mechanical signals of the cell-matrix interaction appear to be the determining factor for the cell fate.

Using micropatterned islands of fibronectin to control cell spreading, it appears that cell shape controls the osteogenic-adipogenic lineage commitment of MSCs through a RhoA-dependent acto-myosin contractility [2] . Adipogenesis is induced in MSCs confined to small ECM islands, while osteogenesis is induced in MSCs, with extensive spreading and higher cytoskeletal tension, on large ECM islands. Inhibition of actin polymerization and consequently tension by cytochalasin $\mathrm{D}$ or inhibition of ROCK activity mimics the phenotype of poorly spread cells, resulting in adipogenesis. Similarly, the myogenicchondrogenic lineage commitment in the transforming growth factor-beta-induced human MSC differentiation is correlated with Rac1 activation. Rac1 activity is significantly higher in smooth muscle cells but lower in chondrogenic differentiation [64]. Using another approach by inhibiting the RhoA/ROCKII pathways with pharmaceuticals such as Y27632, cytochalasin D and jasplakinolide (to stabilize actin polymerization), Woods and colleagues demonstrated that the inhibition of RhoA/ROCK signaling promotes the chondrogenic differentiation of murine embryonic mesenchymal cells via increased Sox 9 expression [65]. These results indicate that cytoskeletal tension and RhoA activity regulate lineage commitment in human MSCs.

Other recent studies also reinforce the importance of RhoA in stem cell differentiation not only for mesodermal stem cells, but also for stem cells from other germ layers such as the ectoderm layer. For example, the response of mammary progenitor cells to substrate rigidity has been demonstrated to be dependent on RhoA activity and cytoskeletal contractility [66]. Linage commitment of neural stem cells depends on substrate rigidity sensing through Rho GTPase. On a stiff ECM substrate, activation of RhoA and Cdc42 suppresses neurogenesis in the neural stem cells; however, the neurogenesis can be rescued by the inhibition of RhoA and Cdc42 [67].

Differentiation of stem cells by fluid flow and mechanical stretching also involves RhoA as part of the cellular sensing mechanism. When fluid flow is applied to C3H10T1/2 murine MSCs, RhoA and ROCKII are activated, which enhances Runx2 expression for commitment to osteogenesis and negatively regulates adipogenic and chondrogenic differentiation [68]. Interestingly, in another study on the effect of continuous cyclic mechanical tension loading on human bone marrow stem cells and $\mathrm{C} 3 \mathrm{H} 10 \mathrm{~T} 1 / 2$ murine MSCs, mechanical tension causes a decrease in RhoA activity, resulting in inhibited Runx2 expression. Pretreatment of the murine stem cells with lysophosphatidic acid restores the alkaline phosphatase and Runx2 expression [69]. These findings indicate that the osteogenic differentiation of MSCs can be regulated by adjusting the method and magnitude of the applied force, consequently activating or deactivating the RhoA signaling pathway that governs the linage commitment.

Rho signals can be activated or inhibited by specific growth factors. In a study with Swiss3T3 fibroblasts, transforming growth factor-beta signals activate RhoA and $\mathrm{RhoB}$, and subsequently induce phosphorylation of LIM domain kinase- 2 and cofilin during actin reorganization. Meanwhile bone morphogenetic protein-7 signals activate RhoA and RhoB, and subsequently induce phosphorylation of ROCK1 but not LIM domain kinase-1/2 [70]. When McBeath and colleagues transfected a dominant-negative RhoA to human MSCs, adipogenesis could be induced even in osteogenic medium. On the contrary, using constitutively active RhoA triggers osteogenesis in adipogenic medium [2]. Hence, RhoA activity appears to be a potential convergence point for mechanical and soluble factor signaling (such as transforming growth factor-beta and bone morphogenetic protein-7) in the control of stem cell differentiation.

In summary, mechanotransduction via the Rho signaling pathway in adult stem cell differentiation has been extensively studied (Figure 2Bii). The signals could be induced by application of mechanical forces, or direct activation or inhibition of the component in the Rho pathways, such as Rho or ROCK. Even though the methodologies in manipulating the mechanical signal or Rho expression varied, the results of the different groups are in agreement. Mechanical forces such as rigid substrate and increased cell spreading (as well as biochemical signals, in some cases) activate RhoA, which will lead to increased cell contractility and bone morphogenetic protein (BMP)-dependent SMA/mothers 
against decapentaplegic (Smad) phosphorylation [71] and/or the activation of pERK, which activates Runx2 [68], and ultimately enhancing osteogenic differentiation. On the contrary, inhibition of Rho/ROCK signaling will lead to the activation of PPAR $\gamma$ or Sox9, enhancing the adipogenic or chondrogenic differentiation, respectively. However, the details of the pathway have yet to be investigated. A number of studies have also demonstrated biophysically induced neuronal differentiation of adult stem cells. The inhibition of RhoA and Rac could rescue the neuronal differentiation on rigid substrates. However, the mechanistic understanding of the mechanotransduction signaling pathway of the neuronal differentiation remains unclear. In addition, although various reports on topography-induced differentiation have suggested the significance of Rho/ROCK signaling and FA activation, the key steps of the mechanotransduction have also yet to be discovered.

\section{Nucleus: mechanical manipulation affects gene regulation}

The intricate physical network described above sets the framework for the physical continuity spanning from the ECM to the nucleus. Mechanical signals such as topographical perturbations from the ECM can be transduced by structural alterations in the network to elicit differential gene expression in stem cells. (See Wang and colleagues and Dahl and colleagues [72,73] for in-depth review of nuclear regulation from mechanotransduction, see Kadrmas and Beckerle [74] for a review of the LIM domain proteins that link the cytoskeleton signal to the nucleus, see Mattout and Meshorer [75] for review of the chromatin plasticity and laminar proteins, and see Shivashankar [76] for a recent review of the nuclear mechanism of mechanosignaling.) We will briefly describe the key components in nuclear mechanotransduction that might play important roles in mechanosensing in stem cells (Figure 2C).

While the actin microfilaments are anchored to the ECM through the integrins, they are also physically connected to the nuclear membrane in a coherent actomyosin cytoskeleton. Nesprins are a class of large outer nuclear membrane proteins that bind actin microfilaments through their KASH domains [77,78]. The KASH domains are then physically connected to the SUN domain protein of the inner nuclear membrane forming a $\mathrm{KASH} / \mathrm{SUN}$ complex to mechanically bridge the actin stress fibers to the nuclear membrane lamins.

A nuclear structure that appears to be important in mechanotransduction is the nuclear lamina. Structurally similar to the cytoskeleton, the nuclear lamina consists of a meshwork of intermediate filaments and lamin proteins that is physically associated with both the KASH/SUN complex $[79,80]$ and chromatin (reviewed in $[72,81]$ ).
Recently, the linkage between nucleoskeleton and cytoskeleton (LINC) complex, a specialized structure that includes the nuclear lamin and SUN which binds to the KASH domain of the actin-associated Nesprins, was identified to link both the nucleoskeleton and cytoskeleton. The existence of the linker of nucleoskeleton and cytoskeleton complex provides more evidence that the mechanical forces arising due to matrix nanotopography and rigidity can physically affect the structural organization of the nucleus [78,82], possibly resulting in altered gene expression. Forces that are transmitted to the nuclear scaffolds via the linker of nucleoskeleton and cytoskeleton complex may regulate critical DNA enzymes or factors. Furthermore, in an earlier study, the disruption of intermediate filaments led to the mechanical decoupling of the integrins and nuclei [83], demonstrating that a direct physical connection exists between the two. Local forces applied to apical integrins have also been shown to transmit to the basal FAs and the nucleus, suggesting that a physical continuity does exist between the ECM and the cell nucleus [84,85].

The nuclei of pluripotent ESCs or adult stem cells exhibit different physical properties compared with nuclei of differentiated cells. Using a micromanipulation method, Pajerowski and colleagues have shown that nuclei in human ESCs are highly deformable, and the nuclei become sixfold stiffer upon differentiation [86]. While the rheological character of the nucleus is set by the nucleoplasm or chromatin, the lack of lamin $\mathrm{A} / \mathrm{C}$ in human ESCs and adult hematopoietic stem cells allows more nuclear deformability. Upon differentiation, nuclei in mouse ESCs start to show the signature of a prestressed nucleus [87]. There is a direct correlation between chromatin assembly and the onset of differentiation in mouse ESCs [88]. The nuclear pore complex composition could also regulate the myogenic differentiation of mouse ESCs [89]. This regulation is evident in the induction of the transmembrane nucleoporin Nup210 during the differentiation from proliferating myoblast and ESCs. Nup210 may thus be required for the induction of genes essential for cell differentiation.

Evidence of nuclear mechanical regulation is also observed in adult stem cells when external force is applied. Other research groups and our group similarly observe nuclear shape changes and altered gene expression in response to topography $[4,90,91]$. While Dalby and colleagues observe spatial alteration of chromosomes in fibroblasts under topographical influence [90], our work involving human MSCs on nanogratings suggests that topography may exert an effect on the structural organization of the nucleus as indicated by the alignment and elongation of the MSC nuclei [4]. In addition to the morphological changes of nuclei, $\mathrm{Li}$ and colleagues also observed a decrease in histone deacetylase activity in 
human MSCs on microgrooves. Compression or stretch was applied to cells on the micropatterns. Forces applied perpendicular to the microgrooves caused a decrease in histone deacetylase, accompanied by an increase in histone acetylation [91]. These studies suggest a direct mechanical coupling of chromatin to the ECM through the intricate mechanotransduction network in stem cells. This physical coupling may allow chromatin regulation through indirect effects of mechanical forces on the exposure of DNA regulatory motifs for transcription factor binding, through the activity of mechanosensitive proteins.

Mechanosensing of ECM rigidity and cell shape can also be transmitted to the nucleus by regulating the transcriptional factors yorkie-homologs Yes-associated protein and transcriptional coactivator with PDZ-binding motif. The regulation of these factors requires Rho GTPase activity and tension in the actomyosin cytoskeleton, but it is independent of the Hippo/LAST cascade. Yes-associated protein/transcriptional coactivator with PDZ-binding motif is required for survival of endothelial cells regulated by cell geometry and, more pertinently, for differentiation of MSCs induced by ECM stiffness. Taken together, the evidence indicates that biophysical signals are transduced into the nucleus both by soluble regulatory factors through nuclear pores and by active stresses through prestressed cytoplasmicnuclear links.

Although there is clear evidence of nuclear mechanotransduction, the molecular and biophysical bases for such mechanisms are still not understood. Nuclear mechanotransduction is currently an area of active research, and a more elaborate discussion can be obtained from [76].

\section{Mechanotransduction among various types of stem cells}

In this review, we have discussed several key signaling components involved in the mechanically induced differentiation, including FA signaling, cytoskeletal contractility, Rho signaling and nuclear regulation, and the recent developments in force-dependent stem cell differentiation. In a collective effort, many groups have been trying to reveal the influence and the mechanism of the biophysical regulation in stem cells using different types of stem cells. A summary of selected recent examples is shown in Table 1.

The majority of the work done to investigate the biomechanical-induced differentiation used adult stem cells as their model systems. MSCs, mainly human, rat and mouse, have been extensively studied. Regardless of the species, the responses are similar. Strong mechanical stimuli such as rigid substrate, increased cell spreading and applied force can stimulate osteogenesis, possibly by increasing actomyosin contractility, activation of RhoA and subsequently Runx2 activity; whereas inhibition of RhoA will result in chondrogenesis or adipogenesis. Applied mechanical force can also induce myogenesis or smooth muscle cell differentiation, requiring either an intermediate substrate rigidity, the activation of Rac1 by cell shape or a combination of growth factors. The use of a soft substrate, anisotropic rigidity, or the inhibition of RhoA and Cdc42 on rigid substrate will promote neuronal differentiation in MSCs, neural stem cells or neural progenitor cells [92].

ASCs are another type of adult stem cell that are promising in clinical applications, although they are not discussed in detail in this review because the mechanically induced differentiation of ASCs has been studied to a lesser extent. Most observations in ASCs are similar to those in MSCs, but some comparisons show a different response to rigidity [46] and increased mechanosensitivity of ASCs. While the inhibition of Rac1 in ASCs enhanced chondrogenesis, which is similar to MSCs, the inhibition of RhoA in ASCs induced alkaline phosphatase expression, indicative of osteogenic differentiation [93]. Nonetheless, further characterization will be needed for ASCs.

ESCs, on the contrary, have different cell characteristics compared with adult stem cells. The majority of the studies have been focused on ESC viability/maintenance, in which inhibition of ROCK activity and actomyosin contractility will increase the viability of the ESCs. Applied forces on undifferentiated ESCs induce spreading. Topography-induced differentiations, however, have been observed in ESCs and MSCs. Anisotropic grating topography can induce neuronal differentiation in ESCs [94] and MSCs [4], while pillar topography can induce osteogenic differentiation in ESCs [95] and MSCs [96]. Taken together, one may speculate that topography activates similar mechanotransduction pathways in the differentiation of ESCs and adult MSCs.

While observations have consistently shown the significance of mechanical signal in stem cell differentiation, the key parameter found in the extracellular niche that activates mechanotransduction is under active research. Despite a large number of studies having demonstrated the effect of substrate rigidity on stem cell differentiation, especially with polyacrylamide hydrogel substrate, a recent paper is challenging this convention. Trappmann and colleagues have shown that spatial control of the cell-matrix interaction, in this case through controlling collagen binding density, could be a major determining factor in stem cell differentiation, instead of substrate rigidity [97]. With the groundbreaking evidence, and other observations on topographyinduced differentiation and the effect of FA-binding site density [20], we believe that the combination of spatial 
Table 1. Summary of stem cell responses to various mechanical stimuli

\begin{tabular}{|c|c|c|c|}
\hline Cell type & Response/regulation & Mechanical stimuli & References \\
\hline \multicolumn{4}{|l|}{ Adult stem cells } \\
\hline \multirow[t]{14}{*}{ MSCs } & Osteogenic differentiation & Applied forces (cyclic/static) & [69] \\
\hline & & Increase cell area & [2] \\
\hline & & Increase in substrate rigidity & [1] \\
\hline & & Activate RhoA & {$[68,69]$} \\
\hline & & Random nanotopography & [96] \\
\hline & Chondrogenic/adipogenic differentiation & Decrease cell area & {$[2,64]$} \\
\hline & & Decrease in substrate rigidity & [1] \\
\hline & & Inhibition of RhoA & [65] \\
\hline & Myogenic/smooth muscle cell differentiation & Applied force (for example, cyclic strain) & [98] \\
\hline & & Intermediate substrate rigidity & [46] \\
\hline & & Cell shape: activation of Rac1 & [64] \\
\hline & Neurogenesis & Soft substrate rigidity & [1] \\
\hline & & Anisotropic (line) topography & [4] \\
\hline & Enhanced endocytosis & Pillar topography & [99] \\
\hline \multirow[t]{2}{*}{ ASCS } & Myogenic differentiation & Intermediate substrate rigidity & [46] \\
\hline & Chondrogenic differentiation & RhoA inhibitor & [93] \\
\hline \multirow[t]{2}{*}{ NSCs/NPCs } & Support neuronal differentiation & Suppression of RhoA and Cdc 42 activity & [67] \\
\hline & & Anisotropic topography & [92] \\
\hline \multirow[t]{4}{*}{ Embryonic stem cells } & Increase spreading & Applied forces & [48] \\
\hline & Increase viability upon dissociation & Inhibition of ROCK activity or actomyosin contractility & {$[60,61]$} \\
\hline & Neuronal differentiation & Anisotropic (line) topography & [94] \\
\hline & Osteogenic differentiation & Pillar topography & [95] \\
\hline
\end{tabular}

ACS, adipose-derived stem cell; MSC, mesenchymal stem cell; NPC, neural progenitor cell; NSC, neural stem cell; ROCK, Rho-associated kinase.

sensing properties (ECM density, geometry, topography) could be as important as, if not more important than, the material properties (rigidity and surface chemistry) of the extracellular environment in inducing the mechanotransduction. Nonetheless, spatial control and material properties will have to be carefully designed to work together in orchestrating signal transduction in the stem cell niche.

\section{Conclusion}

Stem cell differentiation can be regulated by biophysical signals in forms of externally applied forces and/or the manipulation of the substrate rigidity, topography or geometry of ECM patterning. The application of the biophysical signals is sufficient to direct the stem cell fate using minimal or suboptimal biochemical induction, but the biophysical induction can also work in synergy with soluble biochemical cues. The identification of an optimal biophysical environment will therefore be crucial in the fundamental understanding of stem cell differentiation, as well as the application of stem cells in regenerative medicine and cell therapy. The underlying mechanism of the force-mediated differentiation remains unclear. However, recent studies have demonstrated that mechanotransduction through FAs, cytoskeletal contractility, Rho GTPase signaling and the subsequent nuclear regulation are playing major roles in stem cell differentiation. Those components are only part of the complex process of mechanotransduction. Moreover, the interplay between the biochemical signaling pathways and the forcemediated signaling pathways in stem cell differentiation has yet to be discovered. Further investigation will be needed to provide a clearer understanding of biophysically induced stem cell differentiation.

\footnotetext{
This article is part of a thematic series on Physical influences on stem cells edited by Gordana Vunjak-Novakovic. Other articles in the series can be found online at http://stemcellres.com/series/physical
}

\footnotetext{
Abbreviations

ASC, adipose-derived stem cell; ECM, extracellular matrix; ESC, embryonic stem cell; FA, focal adhesion; FAK, focal adhesion kinase; MSC, mesenchymal stem cell; RGD, Arg-Gly-Asp; ROCK, Rho-associated kinase; siRNA, small interfering DNA.
} 


\section{Competing interests}

The authors declare that they have no competing interests.

\section{Authors' contributions}

EKFY and MPS drafted, read and approved the final manuscript.

\section{Acknowledgements}

The authors thank Mechanobiology Institute Singapore, a Research Center of Excellence funded by the Singapore National Research Foundation, for funding support.

\section{Author details}

'Mechanobiology Institute Singapore, National University of Singapore, T-Lab, \#05-01, 5 A Engineering Drive 1, Singapore 117411, Singapore. ${ }^{2}$ Department of Bioengineering, National University of Singapore, EA-03-12, 9 Engineering Drive 1, Singapore 117576. ${ }^{3}$ Department of Surgery, National University of Singapore, NUHS Tower Block, Level 8, 1E Kent Ridge Road, Singapore 119228. ${ }^{4}$ Department of Biological Sciences, Columbia University,1212 Amsterdam Ave, New York, NY 10027, USA.

Published: 31 October 2012

\section{References}

1. Engler AJ, Sen S, Sweeney HL, Discher DE: Matrix elasticity directs stem cell lineage specification. Cell 2006, 126:677-689.

2. McBeath R, Pirone DM, Nelson CM, Bhadriraju K, Chen CS: Cell shape, cytoskeletal tension, and RhoA regulate stem cell lineage commitment. Dev Cell 2004, 6:483-495.

3. McMurray RJ, Gadegaard N, Tsimbouri PM, Burgess KV, McNamara LE, Tare R, Murawski K, Kingham E, Oreffo ROC, Dalby MJ: Nanoscale surfaces for the long-term maintenance of mesenchymal stem cell phenotype and multipotency. Nat Mater 2011, 10:637-644.

4. Yim EK, Pang SW, Leong KW: Synthetic nanostructures inducing differentiation of human mesenchymal stem cells into neuronal lineage. Exp Cell Res 2007, 313:1820-1829.

5. Dado D, Sagi M, Levenberg S, Zemel A: Mechanical control of stem cell differentiation. Regen Med 2012, 7:101-116.

6. Geiger B, Spatz JP, Bershadsky AD: Environmental sensing through focal adhesions. Nat Rev Mol Cell Biol 2009, 10:21-33.

7. Dalby MJ, Childs S, Riehle MO, Johnstone HJ, Affrossman S, Curtis AS: Fibroblast reaction to island topography: changes in cytoskeleton and morphology with time. Biomaterials 2003, 24:927-935.

8. Riveline D, Zamir E, Balaban NQ, Schwarz US, Ishizaki T, Narumiya S, Kam Z, Geiger B, Bershadsky AD: Focal contacts as mechanosensors: externally applied local mechanical force induces growth of focal contacts by an mDia1-dependent and ROCK-independent mechanism. J Cell Bio/ 2001, 153:1175-1186

9. Kim TJ, Seong J, Ouyang M, Sun J, Lu S, Jun PH, Wang N, Wang Y: Substrate rigidity regulates $\mathrm{Ca}^{2+}$ oscillation via RhoA pathway in stem cells. J Cell Physiol 2009, 218:285-293.

10. Kung C: A possible unifying principle for mechanosensation. Nature 2005 , 436:647-654.

11. Reilly GC, Engler AJ: Intrinsic extracellular matrix properties regulate stem cell differentiation. J Biomech 2010, 43:55-62.

12. Guilak F, Cohen DM, Estes BT, Gimble JM, Liedtke W, Chen CS: Control of stem cell fate by physical interactions with the extracellular matrix. Cell Stem Cell 2009, 5:17-26.

13. Teo BKK, Ankam S, Chan LY, Yim EKF: Nanotopography/mechanical induction of stem-cell differentiation. Method Cell Biology 2010, 98:241-294.

14. Vogel V, Sheetz M: Local force and geometry sensing regulate cell functions. Nat Rev Mol Cell Biol 2006, 7:265-275

15. Vogel V, Sheetz MP: Cell fate regulation by coupling mechanical cycles to biochemical signaling pathways. Curr Opin Cell Biol 2009, 21:38-46.

16. Zamir E, Geiger B: Molecular complexity and dynamics of cell-matrix adhesions. J Cell Sci 2001, 114:3583-3590.

17. Ruoslahti E, Obrink B: Common principles in cell adhesion. Exp Cell Res 1996, 227:1-11

18. Lutolf MP, Hubbell JA: Synthetic biomaterials as instructive extracellular microenvironments for morphogenesis in tissue engineering. Nat Biotechnol 2005, 23:47-55.

19. Arnold M, Cavalcanti-Adam EA, Glass R, Blummel J, Eck W, Kantlehner M,
Kessler $\mathrm{H}$, Spatz JP: Activation of integrin function by nanopatterned adhesive interfaces. Chemphyschem 2004, 5:383-388.

20. Schvartzman M, Palma M, Sable J, Abramson J, Hu X, Sheetz MP, Wind SJ: Nanolithographic control of the spatial organization of cellular adhesion receptors at the single-molecule level. Nano Lett 2011, 11:1306-1312.

21. Arnold M, Hirschfeld-Warneken VC, Lohmuller T, Heil P, Blummel J, CavalcantiAdam EA, Lopez-Garcia M, Walther P, Kessler H, Geiger B, Spatz JP: Induction of cell polarization and migration by a gradient of nanoscale variations in adhesive ligand spacing. Nano Lett 2008, 8:2063-2069.

22. Jiang F, Horber $\mathrm{H}$, Howard J, Muller DJ: Assembly of collagen into microribbons: effects of pH and electrolytes. J Struct Biol 2004, 148:268-278.

23. Little WC, Smith ML, Ebneter U, Vogel V: Assay to mechanically tune and optically probe fibrillar fibronectin conformations from fully relaxed to breakage. Matrix Bio/ 2008, 27:451-461.

24. Smith ML, Gourdon D, Little WC, Kubow KE, Eguiluz RA, Luna-Morris S, Vogel $V$ : Force-induced unfolding of fibronectin in the extracellular matrix of living cells. PLoS Biol 2007, 5:e268,

25. Campos LS: Beta1 integrins and neural stem cells: making sense of the extracellular environment. Bioessays 2005, 27:698-707.

26. Zaidel-Bar R, Itzkovitz S, Ma'ayan A, lyengar R, Geiger B: Functional atlas of the integrin adhesome. Nat Cell Biol 2007, 9:858-867.

27. Burridge K, Fath K, Kelly T, Nuckolls G, Turner C: Focal adhesions: transmembrane junctions between the extracellular matrix and the cytoskeleton. Annu Rev Cell Biol 1988, 4:487-525.

28. Geiger B, Bershadsky A, Pankov R, Yamada KM: Transmembrane crosstalk between the extracellular matrix - cytoskeleton crosstalk. Nat Rev Mol Cell Biol 2001, 2:793-805.

29. Gingras AR, Bate N, Goult BT, Hazelwood L, Canestrelli I, Grossmann JG, Liu H, Putz NS, Roberts GC, Volkmann N, Hanein D, Barsukov IL, Critchley DR: The structure of the C-terminal actin-binding domain of talin. EMBO J 2008, 27:458-469.

30. Choi CK, Vicente-Manzanares M, Zareno J, Whitmore LA, Mogilner A, Horwitz AR: Actin and alpha-actinin orchestrate the assembly and maturation of nascent adhesions in a myosin II motor-independent manner. Nat Cell Biol 2008, 10:1039-1050.

31. Even-Ram S, Doyle AD, Conti MA, Matsumoto K, Adelstein RS, Yamada KM: Myosin IIA regulates cell motility and actomyosin-microtubule crosstalk. Nat Cell Biol 2007, 9:299-309.

32. Kanchanawong P, Shtengel G, Pasapera AM, Ramko EB, Davidson MW, Hess $\mathrm{HF}$, Waterman CM: Nanoscale architecture of integrin-based cell adhesions. Nature 2010, 468:580-584

33. Humphries JD, Wang P, Streuli C, Geiger B, Humphries MJ, Ballestrem C: Vinculin controls focal adhesion formation by direct interactions with talin and actin. J Cell Biol 2007, 179:1043-1057.

34. Gingras AR, Vogel KP, Steinhoff HJ, Ziegler WH, Patel B, Emsley J, Critchley DR, Roberts GC, Barsukov IL: Structural and dynamic characterization of a vinculin binding site in the talin rod. Biochemistry 2006, 45:1805-1817.

35. del Rio A, Perez-Jimenez R, Liu R, Roca-Cusachs P, Fernandez JM, Sheetz MP: Stretching single talin rod molecules activates vinculin binding. Science 2009, 323:638-641

36. Garcia AJ, Vega MD, Boettiger D: Modulation of cell proliferation and differentiation through substrate-dependent changes in fibronectin conformation. Mol Biol Cell 1999, 10:785-798.

37. Sawada Y, Tamada M, Dubin-Thaler BJ, Cherniavskaya O, Sakai R, Tanaka S, Sheetz MP: Force sensing by mechanical extension of the Src family kinase substrate p130Cas. Cell 2006, 127:1015-1026.

38. Prager-Khoutorsky M, Lichtenstein A, Krishnan R, Rajendran K, Mayo A, Kam Z, Geiger B, Bershadsky AD: Fibroblast polarization is a matrix-rigiditydependent process controlled by focal adhesion mechanosensing. Nat Cell Biol 2011, 13:1457-1465.

39. Seo CH, Furukawa K, Montagne K, Jeong H, Ushida T: The effect of substrate microtopography on focal adhesion maturation and actin organization via the RhoA/ROCK pathway. Biomaterials 2011, 32:9568-9575.

40. Yim EKF, Darling EM, Kulangara K, Guilak F, Leong KW: Nanotopographyinduced changes in focal adhesions, cytoskeletal organization, and mechanical properties of human mesenchymal stem cells. Biomaterials 2010, 31:1299-1306.

41. Giannone G, Dubin-Thaler BJ, Döbereiner HG, Kieffer N, Bresnick AR, Sheetz MP: Periodic lamellipodial contractions correlate with rearward actin waves. Cell 2004, 116:431-443.

42. Shi P, Shen K, Ghassemi S, Hone J, Kam LC: Dynamic force generation by 
neural stem cells. Cell Mol Bioeng 2009, 2:464-474

43. Galbraith CG, Yamada KM, Sheetz MP: The relationship between force and focal complex development. J Cell Biol 2002, 159:695-705.

44. Yu CH, Law JBK, Suryana M, Low HY, Sheetz MP: Early integrin binding to Arg-Gly-Asp peptide activates actin polymerization and contractile movement that stimulates outward translocation. Proc Natl Acad Sci U S A 2011, 108:20585-20590.

45. Fujita S, Ohshima M, Iwata H: Time-lapse observation of cell alignment on nanogrooved patterns. J R Soc Interface 2009, 6(Suppl 3):S269-S277.

46. Choi YS, Vincent LG, Lee AR, Dobke MK, Engler AJ: Mechanical derivation of functional myotubes from adipose-derived stem cells. Biomaterials 2012 . 33:2482-2491

47. Gerecht S, Bettinger CJ, Zhang Z, Borenstein JT, Vunjak-Novakovic G, Langer $R$ : The effect of actin disrupting agents on contact guidance of human embryonic stem cells. Biomaterials 2007, 28:4068-4077.

48. Chowdhury F, Na S, Li D, Poh YC, Tanaka TS, Wang F, Wang N: Material properties of the cell dictate stress-induced spreading and differentiation in embryonic stem cells. Nat Mater 2010, 9:82-88.

49. Poh YC, Chowdhury F, Tanaka TS, Wang N: Embryonic stem cells do not stiffen on rigid substrates. Biophys J 2010, 99:L19-L21. A published erratum appears in Biophys $J$ 2011, 100:268.

50. Galbraith CG, Yamada KM, Sheetz MP: The relationship between force and focal complex development. J Cell Bio/ 2002, 159:695-705.

51. Vicente-Manzanares M, Ma X, Adelstein RS, Horwitz AR: Non-muscle myosin Il takes centre stage in cell adhesion and migration. Nat Rev Mol Cell Biol 2009, 10:778-790.

52. Etienne-Manneville S, Hall A: Rho GTPases in cell biology. Nature 2002, 420:629-635.

53. Paszek MJ, Zahir N, Johnson KR, Lakins JN, Rozenberg GI, Gefen A, ReinhartKing CA, Margulies SS, Dembo M, Boettiger D, Hammer DA, Weaver VM: Tensional homeostasis and the malignant phenotype. Cancer Cell 2005, 8:241-254.

54. Kimura K, Ito M, Amano M, Chihara K, Fukata Y, Nakafuku M, Yamamori B, Feng J, Nakano T, Okawa K, Iwamatsu A, Kaibuchi K: Regulation of myosin phosphatase by Rho and Rho-associated kinase (Rho-kinase). Science 1996, 273:245-248.

55. Chrzanowska-Wodnicka M, Burridge K: Rho-stimulated contractility drives the formation of stress fibers and focal adhesions. J Cell Biol 1996, 133:1403-1415.

56. Hall A: Rho GTPases and the actin cytoskeleton. Science 1998, 279:509-514.

57. Thomson JA: Embryonic stem cell lines derived from human blastocysts. Science 1998, 282:1145-1147.

58. Zhang L, Valdez JM, Zhang B, Wei L, Chang J, Xin L: ROCK inhibitor Y-27632 suppresses dissociation-induced apoptosis of murine prostate stem/ progenitor cells and increases their cloning efficiency. PLOS ONE 2011, 6:e18271.

59. Chen G, Hou Z, Gulbranson DR, Thomson JA: Actin-myosin contractility is responsible for the reduced viability of dissociated human embryonic stem cells. Cell Stem Cell 2010, 7:240-248.

60. Ohgushi M, Matsumura M, Eiraku M, Murakami K, Aramaki T, Nishiyama A, Muguruma K, Nakano T, Suga H, Ueno M, Ishizaki T, Suemori H, Narumiya S, Niwa H, Sasai Y: Molecular pathway and cell state responsible for dissociation-induced apoptosis in human pluripotent stem cells. Cell Stem Cell 2010, 7:225-239

61. Watanabe K, Ueno M, Kamiya D, Nishiyama A, Matsumura M, Wataya T, Takahashi JB, Nishikawa S, Nishikawa SI, Muguruma K, Sasai Y: A ROCK inhibitor permits survival of dissociated human embryonic stem cells. Nat Biotechnol 2007, 25:681-686.

62. Laplante I, Paquin J, Béliveau R: RhoB expression is induced after the transient upregulation of RhoA and Cdc42 during neuronal differentiation and influenced by culture substratum and microtubule integrity. Dev Brain Res 2001, 129:157-168.

63. Laplante I, Béliveau R, Paquin J: RhoA/ROCK and Cdc42 regulate cell-cell contact and $\mathrm{N}$-cadherin protein level during neurodetermination of $\mathrm{P} 19$ embryonal stem cells. J Neurobiol 2004, 60:289-307.

64. Gao L, McBeath R, Chen CS: Stem cell shape regulates a chondrogenic versus myogenic fate through rac1 and N-cadherin. Stem Cells 2010 28:564-572.

65. Woods A, Wang G, Beier F: RhoA/ROCK signaling regulates Sox9 expression and actin organization during chondrogenesis. J Biol Chem 2005, 280:11626-11634
66. Lui C, Lee K, Nelson CM: Matrix compliance and RhoA direct the differentiation of mammary progenitor cells. Biomech Model Mechanobiol 2011:1-9. doi:10.1007/s10237-011-0362-7

67. Keung AJ, De Juan-Pardo EM, Schaffer DV, Kumar S: Rho GTPases mediate the mechanosensitive lineage commitment of neural stem cells. Stem Cells 2011, 29:1886-1897.

68. Arnsdorf EJ, Tummala P, Kwon RY, Jacobs CR: Mechanically induced osteogenic differentiation - the role of RhoA, ROCKII and cytoskeletal dynamics. J Cell Sci 2009, 122:546-553.

69. Shi Y, Li H, Zhang X, Fu Y, Huang Y, Lui PPY, Tang T, Dai K: Continuous cyclic mechanical tension inhibited Runx2 expression in mesenchymal stem cells through RhoA-ERK1/2 pathway. J Cell Physio/ 2011, 226:2159-2169.

70. Konstantinidis G, Moustakas A, Stournaras C: Regulation of myosin light chain function by BMP signaling controls actin cytoskeleton remodeling. Cell Physiol Biochem 2011, 28:1031-1044.

71. Wang YK, Yu X, Cohen DM, Wozniak MA, Yang MT, Gao L, Eyckmans J, Chen $\mathrm{CS}$ : Bone morphogenetic protein-2-induced signaling and osteogenesis is regulated by cell shape, RhoA/ROCK, and cytoskeletal tension. Stem Cells Dev 2012, 21:1176-1186.

72. Wang N, Tytell JD, Ingber DE: Mechanotransduction at a distance: mechanically coupling the extracellular matrix with the nucleus. Nat Rev Mol Cell Biol 2009, 10:75-82.

73. Dahl KN, Ribeiro AJS, Lammerding J: Nuclear shape, mechanics, and mechanotransduction. Circ Res 2008, 102:1307-1318.

74. Kadrmas JL, Beckerle MC: The LIM domain: from the cytoskeleton to the nucleus. Nat Rev Mol Cell Biol 2004, 5:920-931.

75. Mattout $\mathrm{A}$, Meshorer $\mathrm{E}$ : Chromatin plasticity and genome organization in pluripotent embryonic stem cells. Curr Opin Cell Biol 2010, 22:334-341.

76. Shivashankar GV: Mechanosignaling to the cell nucleus and gene regulation. Annu Rev Biophysics 2012, 40:361-378.

77. Z Zhang Q, Skepper JN, Yang F, Davies JD, Hegyi L, Roberts RG, Weissberg PL, Ellis JA, Shanahan CM: Nesprins: a novel family of spectrin-repeatcontaining proteins that localize to the nuclear membrane in multiple tissues. J Cell Sci 2001, 114:4485-4498.

78. Crisp M, Liu Q, Roux K, Rattner JB, Shanahan C, Burke B, Stahl PD, Hodzic D: Coupling of the nucleus and cytoplasm: role of the LINC complex. J Cell Biol 2006, 172:41-53.

79. Alberts B BD, Lewis J, Raff M, Watson J: Molecular Biology of the Cell. New York: Garland Publishing; 1994

80. Dechat T, Pfleghaar K, Sengupta K, Shimi T, Shumaker DK, Solimando L, Goldman RD: Nuclear lamins: major factors in the structural organization and function of the nucleus and chromatin. Genes Dev 2008, 22:832-853.

81. Gieni RS, Hendzel MJ: Mechanotransduction from the ECM to the genome: are the pieces now in place? J Cell Biochem 2008, 104:1964-1987.

82. Fey EG, Wan KM, Penman S: Epithelial cytoskeletal framework and nuclear matrix-intermediate filament scaffold: three-dimensional organization and protein composition. J Cell Biol 1984, 98:1973-1984.

83. Maniotis AJ, Chen CS, Ingber DE: Demonstration of mechanical connections between integrins, cytoskeletal filaments, and nucleoplasm that stabilize nuclear structure. Proc Nat/ Acad Sci U S A 1997, 94:849-854.

84. Hu S, Chen J, Butler JP, Wang N: Prestress mediates force propagation into the nucleus. Biochem Biophys Res Commun 2005, 329:423-428.

85. Hu S, Chen J, Fabry B, Numaguchi Y, Gouldstone A, Ingber DE, Fredberg Jر J Butler JP, Wang N: Intracellular stress tomography reveals stress focusing and structural anisotropy in cytoskeleton of living cells. Am J Physio/ Cell Physiol 2003, 285:C1082-C1090.

86. Pajerowski JD, Dahl KN, Zhong FL, Sammak PJ, Discher DE: Physical plasticity of the nucleus in stem cell differentiation. Proc Natl Acad Sci U S A 2007, 104:15619-15624.

87. Mazumder A, Shivashankar GV: Emergence of a prestressed eukaryotic nucleus during cellular differentiation and development. J R Soc Interface 2010, 7:S321-S330

88. Bhattacharya D, Talwar S, Mazumder A, Shivashankar GV: Spatio-temporal plasticity in chromatin organization in mouse cell differentiation and during Drosophila embryogenesis. Biophys J 2009, 96:3832-3839.

89. D'Angelo M, Gomez-Cavazos JÂ, Mei A, Lackner D, Hetzer M: A change in nuclear pore complex composition regulates cell differentiation. Dev Cell 2012, 22:446-458.

90. Dalby MJ, Gadegaard N, Herzyk P, Sutherland D, Agheli H, Wilkinson CD, Curtis AS: Nanomechanotransduction and interphase nuclear organization influence on genomic control. J Cell Biochem 2007, 102:1234-1244. 
91. Li Y, Chu JS, Kurpinski K, Li X, Bautista DM, Yang L, Paul Sung KL, Li S: Biophysical regulation of histone acetylation in mesenchymal stem cells. Biophys J 2011, 100:1902-1909.

92. Moe AA, Suryana M, Marcy G, Lim SK, Ankam S, Goh JZ, Jin J, Teo BK, Law JB, Low HY, Goh EL, Sheetz MP, Yim EK: Microarray with micro- and nanotopographies enalbes identication of the optimal topography for directing the differentiation of primary murine neural progenitor cells. Small 2012. [Epub ahead of print]

93. Jungmann PM, Mehlhorn AT, Schmal H, Schillers H, Oberleithner H, Südkamp NP: Nanomechanics of human adipose-derived stem cells: Small GTpases impact chondrogenic differentiation. Tissue Eng Part A 2012, 18:1035-1044.

94. Lee MR, Kwon KW, Jung H, Kim HN, Suh KY, Kim K, Kim KS: Direct differentiation of human embryonic stem cells into selective neurons on nanoscale ridge/groove pattern arrays. Biomaterials 2010, 31:4360-4366.

95. Markert LD, Lovmand J, Foss M, Lauridsen RH, Lovmand M, Füchtbauer EM, Füchtbauer A, Wertz K, Besenbacher F, Pedersen FS, Duch M: Identification of distinct topographical surface microstructures favoring either undifferentiated expansion or differentiation of murine embryonic stem cells. Stem Cells Dev 2009, 18:1331-1342.
96. Dalby MJ, Gadegaard N, Tare R, Andar A, Riehle MO, Herzyk P, Wilkinson CDW Oreffo ROC: The control of human mesenchymal cell differentiation using nanoscale symmetry and disorder. Nat Mater 2007, 6:997-1003.

97. Trappmann B, Gautrot JE, Connelly JT, Strange DG, Li Y, Oyen ML, Cohen Stuart MA, Boehm H, Li B, Vogel V, Spatz JP, Watt FM, Huck WT: Extracellularmatrix tethering regulates stem-cell fate. Nat Mater 2012, 11:642-649.

98. Park JS, Chu JSF, Cheng C, Chen F, Chen D, Li S: Differential effects of equiaxial and uniaxial strain on mesenchymal stem cells. Biotechnol Bioeng 2004, 88:359-368.

99. Teo BKK, Goh SH, Kustandi TS, Loh WW, Low HY, Yim EKF: The effect of micro and nanotopography on endocytosis in drug and gene delivery systems. Biomaterials 2011, 32:9866-9875.

doi:10.1186/scrt132

Cite this article as: Yim EKF, Sheetz MP: Force-dependent cell signaling in stem cell differentiation. Stem Cell Research \& Therapy 2012, 3:41. 